\title{
A Cognitive Inference Approach for Developing Medical Diagnostic Expert Systems
}

\author{
Ashish Chandiok \\ Faculty of Engineering \\ Dayalbagh Educational Institute \\ Dayalbagh, Agra, UP, India
}

\author{
D. K. Chaturvedi \\ Faculty of Engineering \\ Dayalbagh Educational Institute \\ Dayalbagh, Agra, UP, India
}

\begin{abstract}
Cognitive approaches are nowadays the most popular and widely used means of computing information due to its human-like sensing, comprehending, and action. The cognitive system can handle diverse data, uses modern technologies like natural language processing, machine learning, semantics, and decision support. It can have human interaction behavior with contextual data handling. The decisions are also not made on fixed rules, but human-like weight based judgments. Expert systems (ES's) are intelligent software tools that use acquired knowledge from experts in a specific domain to offer assistance to its users over a dialog or a query talks conducted between the user and the ES application software. This research work is for implementing a cognitive approach for determining diagnosis decisions providing expert system services. Cognitive strategies frequently denoted as "Human-like intelligent Computing Method" using restricted processing, storing and displaying skills. This paper presents a new diagnosis problem resolving model grounded in the investigation of the relationships among symptoms and illnesses in the form of certainty and severity elements. The article also acquaints with a modified knowledge representation appropriate for a cognitive system with limited structured data handling capabilities using XML language. The proposed model thrived and tested in the domain of medicals like fever (Flu, Pneumonia, and Cold Fever) using real knowledge base acquired by the mayo clinic. The earned consequences establish the strength and competence of the planned model.
\end{abstract}

\section{General Terms}

Cognitive Inference, Diagnostic, Expert system

\section{Keywords}

Cognitive, Expert System tools, Medical Expert Systems, Knowledge Representation, Problem Resolving Modeling

\section{INTRODUCTION}

An expert system (ES) represents knowledge-based intelligent software system that offers assistance to its users over a dialog system or a question answering based dialog, focused on the user and the ES application. Cognitive systems mostly handle unstructured data as a knowledge base. The cognitive approach mainly uses various technologies like natural language processing, machine learning, decision support, computer vision, and speech processing. So, it is giving the systems, with human-like information handling power. The aim of the research work is dual; the first is to inspect the problems that cover the procedure of developing expert system applications. The second one is to present an innovative diagnosis problem resolving technique centered on certainty and severity factors in the medical domain. Some of the prior implementation, directed to building expert systems on traditional rule-based models. The principal drawbacks of these rule-based expert systems are the inability to represent accurately physical world incidences as the knowledge organized in the abstract. Secondly, the ineffectiveness of the rule structure to attain learning from errors. Lastly, the difficulties faced by knowledge engineers to achieve experience from the domain experts regularly. Most of the previous expert systems based on rules remained mostly laboratory research trials that only temporarily achieve success in real-world scenarios. These systems have the problem of designing numerous rules for a particular situation. On the other hand, building expert systems based on machine learning approach also did not show good results. The significant problems with such pragmatic models are as follows. Firstly it provides the challenge of generating and developing a significant amount of feature vector data for training the model. The training done in abstract, so it is not possible to add new parameters online. The training takes a lot of iterations to reach an optimum result. The trained models can take only specific parameters on which the prototype is taught. The work argues that having such system is not suitable for constructing individual expert system software and is far from the reality of having human-like expertize. So, to improve the system, the work proposes to build expert system based on a cognitive approach which has extra advantages of human-like data handling, expert weight on giving decisions multi-criteria decision making and compared to rule-based and machine learning models.

\subsection{Related Works}

In a general definition a Knowledge-Based System (KBS), denoted as an Expert System (ES) is defined as: "intelligent computer software which utilizes knowledge and inference processes to solve problems that are sufficiently hard to need noteworthy human expertise for their result" [13]. The knowledge base and inference engine are two key modules of a Knowledge-based experts system [13, 3]. The progress of KBS and ES was started in early 1970s, especially in the field of medical diagnosis. INTERNIST-I is the expert system designed in the early 1970's to diagnose various dis- 
eases in inner medicine by exhibiting the conduct of doctors. In 1982 , the INTERNIST-I project signified fifteen years of effort, and by particular information enclosed $70-80 \%$ of all the likely diagnoses in case of internal medicine. After few years around 1976 Shortliffe developed MYCIN [25]. MYCIN uses the knowledge base built by a set of rules related to bacterial infection. Originally it has around 500 rules and integrated the backward chaining inference method. In 1980s, MYCIN generalized to EMYCIN [28] as a casing to implement it for constructing further expert systems and thus based on this platform, the researchers created PUFF [1] in the year 1982 as a medical expert system. PUFF interprets measurements from respiratory tests to determine the presence and severity of lung disease in the patient. In [26], about year 1986 Shortliffe elucidates the past of expert systems and preferably signifying medical expert systems. In year 1993, Bonfa et al., designed HERMES [5] as a different medical expert system in the area of hepatic diseases. In the year 1994, Hudson and Cohan built medical expert systems that include the Fuzzy Logic to tackle the ambiguity which pertains due to indefinite or erroneous info [14]. In 2001, Schmidt et al., illuminate regarding Case-based Reasoning (CBR) and its potential use as a reasoning performance in medical expert systems [24]. In 2006, Keleş and Keleş developed ESTDD [16], as an Expert System for Thyroid Diseases Diagnosis. In year 2007, Akbarzadeh-T et al., recommended a universal technique for the taxonomy and diagnosis in medical system and applied it to aphasia diagnosis as an expert system [2]. Also in the year 2009, Kumar et al., exemplified a hybrid methodology using CBR and Rule-based Reasoning for domain free clinical decision support concerning Intensive Care Units (ICU) [18]. In 2011, with the progress of World Wide Web many question answering system developed based on this technology. Başçiftçi et al., develop web-based medical decision support system application for diagnosis of coronary heart disease using the new method of Boolean functions minimization [4]. In 2011, Lee et al., developed a fuzzy ontology-based diagnostic system for curing diabetes [19]. In 2014, Zhang et al., created a pure ontology-based diagnosis system for curing mild cognitive impairment [29]. Also, in [15], Jafarpour et al., developed a semantic web-based clinical practice guideline engine using OWL technologies. In year 2015, Samwald et al., created a method for representing knowledge for pharmacogenomics, with genome base reasoning for clinical decision support system using OWL 2 DL ontologies [23]. In the same year, Chi et al., developed a chronic disease dietary consulting system based on ontologies (OWL) and semantic rules [10]. In [30], the year 2016, Zhang et al., developed and designed a sharable Clinical Decision Support System using third generation web technologies based on semantic web service framework. Apart from Rulebased, Case-based and knowledge representation (Ontologies and Semantic web), another group developed diagnostic inference system using machine learning and computational intelligence models [9]. In 2011, Gagliardi developed Instance-based classifiers and realized it on medical databases to extract knowledge and diagnose medical problems [12]. In 2011, Keleş et al. developed an expert system using the neuro-fuzzy model to diagnose breast cancer [17]. In 2013, Chen et al., developed rule-based decision-making to have a diagnosis system for evaluating arteriovenous shunt stenosis for hemodialysis cure of patients using the technology fuzzy Petri nets [8]. In 2013, Stoean, R. and Stoean, C., developed a model for medical decision making by utilizing supervised machine learning technique known as support vector machines, and also extract feature selection using rules of evolutionary algorithms [27]. In 2011, Papageorgiou developed a novel procedure for decisions in medical informatics with the help of fuzzy cognitive maps in combination with fuzzy rule-extraction techniques [22]. In 2014, Douali et al., created a diagnosis support system founded on clinical guidelines and compared outcome using case-based fuzzy cognitive maps with Bayesian networks [11]. In 2014, Zhu et al., created an intelligent system for lung cancer diagnosis using an innovative genetic algorithm based feature choice technique [20]. In 2015, Mohapatra et al., created a better cuckoo search based extreme learning machine model for classifying medical data to diagnose medical problems [21]. Bonze et al., in 2016, developed a sophisticated SNP diseases selecting and organizing by hybrid association rule mining and artificial neural network which is based on evolutionary algorithms [6]. All the past methods and systems for creating medical expert system has the problem of creating abstract knowledge model. These models also lack the human-like multi-criteria decision making. So, for developing a better medical diagnostic expert system and remove the problem of static and abstract knowledge development with human-like cognitive inference, this work proposes a novel architecture model for a diagnostic expert system in continuing work of Chandiok et al., [7].

\subsection{Aim of the Paper}

The primary purpose of the paper is to show by following points:

(1) To review the past medical diagnostic expert system, and discover limits of the previous methods.

(2) To propose a novel architecture for cognitive inference based medical expert system to have a rule and model-free knowledge representation and reasoning.

(3) To implement and evaluate the proposed system for performance.

The paper consists of the following sections. Section 2 explains general expert system. Also, Section 2 proposes the cognitive inference based system for medical diagnosis. Section 3 layouts the method for developing such systems. Section 4 offers the results by evaluating the system performance and also discusses the salient features of such systems. Section 5 is showing the conclusion of the work.

\section{BACKGROUND}

This paper proposes a standalone cognitive inference approach based expert system application that totally implemented on the interactive user-software platform shown in Figure 2. The work discusses the challenges faced and how to deal with them. At the end of our work, we shall conduct a comparison between our approach and other approaches that depend on relaying heavy load on the backend knowledge and just implementing the interface on the system. An expert system is a system that uses human knowledge taken inside a computer to resolve problems that typically need human expertise as shown in Figure 1 The essential constituents that describe expert systems are Knowledge-base, Inference engine, and Working memory. A real physical time expert system application involves two more modules denoted as a database, and Interface. Knowledge-base is the portion of an expert system in which the knowledge attained from its numerous sources saved in the arrangement of rules, frames, logic statements, machine learning models, case-based, soft computing models, semantic models, or any other representation. In a classical expert system application, the Knowledge base may entail colossal storage space, specially in a case based reasoning expert systems that depend on facts in their rationales. 


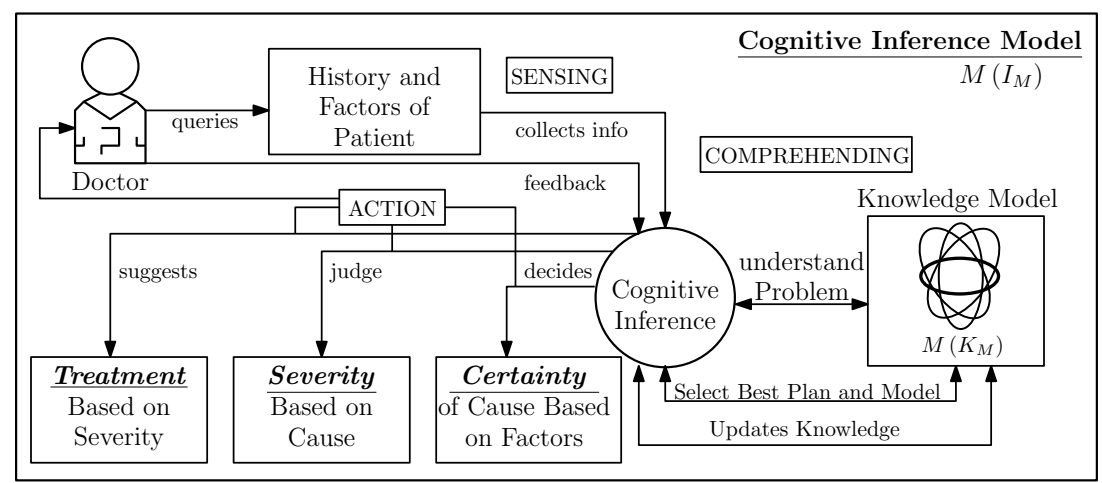

Fig. 2. Proposed Cognitive Inference Model for Medical Diagnosis.



Fig. 1. General Expert System Building Block.

The inference engine is the critical information processing component of the expert system; it chooses the portions of knowledge to be realized and analyzed in the course of its reasoning procedure to derive up with new deductions concerning the existing scenario. It also decides the fragments of info needed from the user to complete its thinking process for making a decision.

The working memory is the part of the expert system that embraces the present values given by the user through the interrogative session, the determined values in the course of the reasoning progression, and the transitional values calculated during the computation and logic.

In real-world scenario for developing the expert system applications the need is to develop cognitive expert system shown in Figure 2. the quantity of raw data required to be delivered by the user is enormous, and the user has to pass in these facts each instance he/she performs a new session with the expert system. So it is practically essential to keep this data in a database as stationary info. An example of such evidence is the information desirable about gas properties for measuring air pollution, soil features for a precise farm, or the past details of a specific patient.

The interface is the audio/visually observable fragment of the expert system where the user can act together with the expert system by giving contextual data and watching outcomes.

For each of the constituents mentioned above, we have precisely two choices: either we implement it on interactive devices like tablets, laptops, or mobile device, or create it on a server. Each of the two options affects the complete enactment of the expert system by accessibility, maintenance, compactness, and upgrades.

\section{METHOD}

\subsection{Understand and classify the question using Natural Language Processing tools (NLP) and Semantic Web (SW) tools:}

Constructing a standalone cognitive approach based expert system application on computer devices will involve the following points:

(1) Expressing knowledge in a simple lightweight, and universal format, appropriate for the limited storage and handling abilities of computing devices.

(2) Constructing a user interface suitable for the human-computer interaction and dealing narrow capabilities of low computing devices in regarding its screen size, and the approach of the data record.

(3) Structuring a custom-built inference engine having the behavior of human-like functionality. It must also fit for the limited handling abilities of the gadgets, using the particular knowledge representation.

(4) Utilization of the expert system application on different hardware platforms under uncertain conditions.

\subsection{Representation of Knowledge}

Currently, XML is chosen and adopted freshly as a representation language for data representation of numerous types of applications for its light-weight; ease of treating data, and for being maintained by almost all other programming languages. It offers a set of rules for generating a hierarchical structure of a useful knowledge document. These structures get nested such that each element built of other features. Factors may include attributes, which are assigned values for quantitative reasoning.

\subsection{Constructing Domain Knowledge}

The domain knowledge contains of two portions: domain ontology and domain models. Domain ontology deliberated as the best essential component of domain knowledge: It describes the semantics of the realm, i.e., the relations used to describe the domain: This language comprises of various constructs like concepts involving concepts, relationships, and expressions. On the other hand, Domain models are comprehensible assemblies of statements regarding the domain that signify specific perspectives on the domain knowledge that is apposite for solving the uncertain problem 
and give a decision. A concept is a dominant construct in domain modeling. It may have an inner structure formed by a set of properties: Every property assigned a single or several values from a given value set. Also, expressions about the domain construct deliver an appropriate way of modeling the arrangement of domain knowledge. In which pure manifestations such as: "Fever: Cough= high" where "Fever" is a concept having the property denoted by "Cough" and "high" is one of the possible values of this property. Both concepts and expressions used for describing a particular set of domain relations: For instance, IF Fever: Cough = "high" AND Fever: Chills = "Yes" AND Fever: a Sore Throat = "Yes" Then Disorder: Name = "Pneumonia."

The domain ontology describes the terms used in the domain and is known as (domain terms), and the knowledge categories that these words have (e.g., concept, attribute, value). The domain model defines different cases of each disorder in the knowledge base.

\subsection{Deployment of the Cognitive Approach based Expert System Application}

The deployment of the application based on $\mathrm{C \#}$ and python. The $\mathrm{C} \#$ is a dominant language having the ability to develop software for desktop, web, and mobiles. The development of the application is through Visual Studio IDE (Integrated Development Environment).

\subsection{Cognitive Approach Based User Interface}

The traditional technique of user interaction inside an expert system depends on constructing a dialog between the user and the expert system. The approach for dialog is a sequence of queries offered to the user one at a time, and conferring to the response delivered by the user to each question, the following query created. The structure of offered queries also known by consulting to the order of the hardcoded set of the rules presents inside the knowledge-base and on the arrangement of inferences in the structure of implementing the task.

Most of the classical knowledge-based system adopts the methodology mentioned above, but it has the various difficulties:

(1) Concentrated to the mentioned approach, the user has to interact with the system for very lengthy sessions, through which he/she is forced to reply a sequence of numerous queries; some of the queries may remove if the questions offered in an altered arrangement.

(2) The user has to reply every query in a direction to acquire the reasoning outcomes. However, occasionally the user might not have the answers to each question presented and needs to resolve to render to the responses that he has previously specified to the system.

(3) At times the queries do not exist logically. The problem occurs because question produced rendering to the way of rules in the knowledge-base, and the order of evidences and arrangements that establish the rules.

(4) The user cannot select the questions for which he knows the answers; he is indulged to trail a series of questions accessible by the system.

(5) The user has no flexibility to go back and forth through these queries, so he can alter the answers that he provides in preceding queries.

(6) The classical method does not permit the user to outlook the reasoning progress and the consequence of his /her responses throughout the interrogation session. Only the concluding outcomes are accessible to the user at the finish of the query session.

In this cognitive approach, a generic user interface created to solve all the problems that occur in the previous classical interfaces for expert systems which depend on its diagnostic model and logic.

\subsection{Diagnosis Problem Solving Model}

Most of the old-style diagnosis problem-solving prototypes implements single-problem supposition - (the system expects to give a single disorder) - that disclose a one to one relationship based causal model giving the conclusion for a different disease based on symptoms.

In this work we shall introduce a cognitive approach based model for solving diagnosis problem bearing in mind multiple-disorder supposition - (where more than one disorder handling expected from the system) - based on the analysis of the degree of certainty and the degree of severity of the suspected disorders.

\subsection{Cognitive Approach to Diagnosis Problem Solving Method}

The diagnosis problem-solving system model aims to decide the disorders that ground the symptoms perceived by the user or the person suffering from illness. But sometimes symptoms of various disorders are alike. So, it creates the judgment of deciding the correct disorder very hard and needs more thorough knowledge to be assimilated from the domain expert to approve the reality of a particular disease and reject other doubted disorders.

Proposed Cognitive Inference approach for solving problem of diagnosis Important terms related to the cognitive inference of multiple input problems.

(1) The set of causes (disorders) the patient is suffering:

$C=\left\{c_{1}, c_{2}, \ldots, c_{n}\right\}$

(2) The group of factors that creates particular Cause: $F(c)=\{f: f$ is a factor of $c, c \in C\}$

(3) The set of all factors that represent the patient illness: $F=\bigcup_{i=1}^{n} F\left(d_{i}\right)$

(4) The set of symptom observed by the user: $S=\{s:$ s is a user observation, $s \in F\}$

(5) The set of suspected causes due to the symptoms found inside the patient

$C_{\text {suspected }}=\{c: c \in C,(S(c) \bigcap F) \neq \phi\}$

(6) The set of expected factors due to alleged cause: $F_{\text {exp ected }}=$ $\left\{f: f \in f(c), c \in C_{\text {suspected }}\right\}$

(7) The certainty of a cause presence obtained by the occurrence of its factors as suspected symptoms. Each factor $\mathrm{f}$ has a participation proportion given by $P(f)$, which is calculated by $\frac{1}{p}$ where $\mathrm{p}$ is the number of causes related to factor f. $P(f)=$ $\frac{1}{p} ; p=$ number of causes related to $f$

(8) Certainty of cause $(c)$ is the max value of the participation proportion of the observation regarding to a particular cause. $C R(c)=\max _{0<k<l} P\left(f_{k}(c)\right)$

(9) Severity of the cause is obtained by the occurrence of its symptoms. Each factor $(f)$ for a cause $(c)$ determines a particular severity confidence level $C(f)$ for a specific cause that may be defined by the domain expert confidence $C f_{s}(f)$ $\forall c \in C, f \in f(d)\left(C f(f)=C f_{s}(f)\right.$ or $\left.\frac{1}{k}, k=|F(c)|\right)$ 
(10) Severity of the cause $C$ Objects cannot be created from editing field codes. is the sum of the confidence of its factors:

$$
S V(c)=\sum_{i=1}^{n} C f\left(f_{i}(c)\right)
$$

\subsection{Algorithm for Diagnostic Model}

Algorithm for Diagnostic Model // Initialize the system considering all Causes as suspected and all factors as expected

$C_{\text {suspected }}=C$

$F_{\text {expected }}=F$

// Initialize No symptoms found in the patient

$S=\{\}$

// Initialize the confidence value of each factor according to particular cause

$\forall c \in C, f \in f(d)\left(C f(f)=C f_{s}(f)\right.$ or $\left.\frac{1}{k}, k=|F(c)|\right)$

// Initialize the participation proportion

$P(f)=\frac{1}{p} ; p=$ number of causes related to $f$

For each Expected Symptom as Observation

\{

$S=S+$ NewSymptom

$C_{\text {suspected }}=\{c: c \in C,(S(c) \bigcap F) \neq \phi\}$

$F_{\text {exp ected }}=\left\{f: f \in f(c), c \in C_{\text {suspected }}\right\}$

// calculate the Severity and Certainty factors

$\forall c \in C, f \in f(d)\left(C f(f)=C f_{s}(f)\right.$ or $\left.\frac{1}{k}, k=|F(c)|\right)$,

$S V(c)=\sum_{i=1}^{n} C f\left(f_{i}(c)\right)$

$P(f)=\frac{1}{p} ; p=$ number of causes related to $f, C R(c)=$ $\max _{0<k<l} P\left(f_{k}(c)\right)$

$0<k<l$

Output $C_{\text {suspected }}$

Output $f_{\text {exp ected }}$

\}

Return $C_{\text {suspected }}$

The algorithm returns the certainty and severity value for each cause according to selected factors after observation of the patient. At the end, the highest value of suspected cause taken as the outcome of patient illness.

\section{RESULT AND DISCUSSION}

Consider Symptom=Fever. Table 1 shows the Factors accompanied by the mention fever. $F=\left\{f_{1}, f_{2}, \ldots, f_{n}\right\}$ Causes are suspected

Table 1. Factors Associated With Symptom Fever

\begin{tabular}{|l|l|l|l|}
\hline Factors & Name & Factors & Name \\
\hline$f_{1}$ & $\begin{array}{l}\text { Extreme Fatigue with } \\
\text { body and Muscle aches }\end{array}$ & $f_{10}$ & High Fever with Chills \\
\hline$f_{2}$ & Headaches & $f_{11}$ & Acting Confused \\
\hline$f_{3}$ & No Appetite & $f_{12}$ & $\begin{array}{l}\text { Coughing and sore } \\
\text { throat }\end{array}$ \\
\hline$f_{4}$ & High Fever & $f_{13}$ & $\begin{array}{l}\text { Stuffy or runny nose and } \\
\text { sneezing }\end{array}$ \\
\hline$f_{5}$ & Chills and Sweats & $f_{14}$ & Eyes that water \\
\hline$f_{6}$ & Stuffy nose and cough & $f_{15}$ & $\begin{array}{l}\text { Some muscle aches and } \\
\text { headaches }\end{array}$ \\
\hline$f_{7}$ & $\begin{array}{l}\text { Cough with mucus or } \\
\text { even blood }\end{array}$ & $f_{16}$ & \begin{tabular}{l} 
Low or No Fever \\
\hline$f_{8}$
\end{tabular} Trouble breathing \\
\hline$f_{9}$ & Chest pain & $f_{17}$ & Coughing \\
\hline
\end{tabular}

These are the factors associated with the fever symptom. when a set of factors observed inside a patient. The knowledge base created for relation between factors as a combination denoted as

$$
C=\left\{c_{1}, c_{2}, \ldots, c_{n}\right\}
$$

Table 2 shows the cause list according to the fever: The knowledge

Table 2. Causes Related to Symptoms Fever

\begin{tabular}{|l|c|}
\hline Cause & Cause Name \\
\hline$c_{1}$ & Influenza(flu) \\
\hline$c_{2}$ & Pneumonia \\
\hline$c_{3}$ & Common Cold \\
\hline \\
These are the causes related with \\
the fever symptom
\end{tabular}

base relating factors to each cause given by:

$F\left(c_{1}\right)=\left\{f_{1}, f_{2}, f_{3}, f_{4}, f_{5}, f_{6}, f_{17}, f_{18}\right\}$

$F\left(c_{2}\right)=\left\{f_{7}, f_{1}, f_{8}, f_{9}, f_{10}, f_{2}, f_{3}, f_{11}, f_{17}\right\}$

$F\left(c_{3}\right)=\left\{f_{12}, f_{13}, f_{14}, f_{15}, f_{16}, f_{17}, f_{18}\right\}$

The knowledge base shows that many factors are common among these causes. So it is not easy for a doctor to diagnose patient. The system based on cognitive approach tries to solve a complex multicriterion diagnostic decision-making problem using the method mention above. Table 3 shows the matrix table gives the factor participation in combination of cause From the participation table of

Table 3. Factors participation in number of causes

\begin{tabular}{|l|l|l|l|}
\hline Factors & Causes & $\begin{array}{l}\text { Number of } \\
\text { Causes that cause } \\
\text { the factor }\end{array}$ & $\begin{array}{l}\text { Participation } \\
\text { of Causes }\end{array}$ \\
\hline$f_{1}$ & $c_{1}, c_{2}$ & 2 & $50 \%$ \\
\hline$f_{2}$ & $c_{1}, c_{2}$ & 2 & $50 \%$ \\
\hline$f_{3}$ & $c_{1}, c_{2}$ & 2 & $50 \%$ \\
\hline$f_{4}$ & $c_{1}$ & 1 & $100 \%$ \\
\hline$f_{5}$ & $c_{1}$ & 1 & $100 \%$ \\
\hline$f_{6}$ & $c_{1}$ & 1 & $100 \%$ \\
\hline$f_{7}$ & $c_{2}$ & 1 & $100 \%$ \\
\hline$f_{8}$ & $c_{2}$ & 1 & $100 \%$ \\
\hline$f_{9}$ & $c_{2}$ & 1 & $100 \%$ \\
\hline$f_{10}$ & $c_{2}$ & 1 & $100 \%$ \\
\hline$f_{11}$ & $c_{2}$ & 1 & $100 \%$ \\
\hline$f_{12}$ & $c_{3}$ & 1 & $100 \%$ \\
\hline$f_{13}$ & $c_{3}$ & 1 & $100 \%$ \\
\hline$f_{14}$ & $c_{3}$ & 1 & $100 \%$ \\
\hline$f_{16}$ & $c_{3}$ & 1 & $33 \%$ \\
\hline$f_{17}$ & $c_{1}, c_{2}, c_{3}$ & 3 & $50 \%$ \\
\hline$f_{18}$ & $c_{1}, c_{3}$ & 2 & $50 \%$ \\
\hline$f_{18}$ & $c_{1}, c_{2}$ & 2 & \\
\hline These are the factors associated with the fever symptom. & & $100 \%$ \\
\hline
\end{tabular}

each factor, the values can help to determine the Certainty of the cause. The cause that occurs with highest certainty factor has the maximum chance to get chosen as the disorder occurring in the patient.

The severity of the illness obtained from the following table 4 in which cause related to the particular symptom is considered necessary.

Consider some test Cases to evaluate the system performance, the following table shows the result: Test Case 1: Considering the patient observed to have $f_{1}, f_{4}, f_{18}$ shown in Table 5 According to the condition above considering the symptoms belonging to all 
Table 4. Confidence Value of Severity According to Each Factor

\begin{tabular}{|l|l|l|l|l|}
\hline Factors & Causes & $\begin{array}{l}\text { Initial } \\
\text { Assign } \\
\text { Confidence }\end{array}$ & $\begin{array}{l}\text { Expert Con- } \\
\text { fidence }\end{array}$ & $\begin{array}{l}\text { Final } \\
\text { Confi- } \\
\text { dence }\end{array}$ \\
\hline$f_{1}$ & $c_{1}$ & 0.12 & 0.7 & 0.7 \\
\hline$f_{2}$ & $c_{1}$ & 0.12 & 0.3 & 0.3 \\
\hline$f_{3}$ & $c_{1}$ & 0.12 & 0.3 & 0.3 \\
\hline$f_{4}$ & $c_{1}$ & 0.12 & 0.6 & 0.6 \\
\hline$f_{5}$ & $c_{1}$ & 0.12 & 0.5 & 0.5 \\
\hline$f_{6}$ & $c_{1}$ & 0.12 & 0.4 & 0.4 \\
\hline$f_{17}$ & $c_{1}$ & 0.12 & 0.2 & 0.2 \\
\hline$f_{18}$ & $c_{1}$ & 0.12 & 0.2 & 0.2 \\
\hline$f_{7}$ & $c_{2}$ & 0.11 & 0.7 & 0.7 \\
\hline$f_{8}$ & $c_{2}$ & 0.11 & 0.5 & 0.5 \\
\hline$f_{9}$ & $c_{2}$ & 0.11 & 0.7 & 0.7 \\
\hline$f_{10}$ & $c_{2}$ & 0.11 & 0.5 & 0.5 \\
\hline$f_{11}$ & $c_{2}$ & 0.11 & 0.6 & 0.6 \\
\hline$f_{1}$ & $c_{2}$ & 0.11 & 0.5 & 0.5 \\
\hline$f_{2}$ & $c_{2}$ & 0.11 & 0.3 & 0.3 \\
\hline$f_{3}$ & $c_{2}$ & 0.11 & 0.3 & 0.3 \\
\hline$f_{17}$ & $c_{2}$ & 0.11 & N/A & 0.11 \\
\hline$f_{12}$ & $c_{3}$ & 0.14 & 0.7 & 0.7 \\
\hline$f_{13}$ & $c_{3}$ & 0.14 & 0.6 & 0.6 \\
\hline$f_{14}$ & $c_{3}$ & 0.14 & 0.6 & 0.6 \\
\hline$f_{15}$ & $c_{3}$ & 0.14 & 0.5 & 0.5 \\
\hline$f_{16}$ & $c_{3}$ & 0.14 & 0.6 & 0.6 \\
\hline$f_{17}$ & $c_{3}$ & 0.14 & 0.3 & 0.3 \\
\hline$f_{18}$ & $c_{3}$ & 0.14 & 0.2 & 0.2 \\
\hline
\end{tabular}

These are the factors associated with the fever symptom.

Table 5. Test Case 1 result:

\begin{tabular}{|c|l|l|l|l|l|l|}
\hline \multirow{2}{*}{ Dataset } & \multicolumn{2}{|c|}{$c_{1}$} & \multicolumn{2}{c|}{$c_{2}$} & \multicolumn{2}{c|}{$c_{3}$} \\
\cline { 2 - 7 } & CER & SEV & CER & SEV & CER & SEV \\
\hline \multirow{2}{*}{$f_{1}, f_{4}, f_{18}$} & $50 \%$ & 1.1 & $50 \%$ & 0.6 & $50 \%$ & 0.2 \\
\hline
\end{tabular}

Table 6. Test Case 2 result:

\begin{tabular}{|l|l|l|l|l|l|l|}
\hline \multirow{2}{*}{ Dataset } & \multicolumn{2}{|c|}{$c_{1}$} & \multicolumn{2}{c|}{$c_{2}$} & \multicolumn{2}{c|}{$c_{3}$} \\
\cline { 2 - 7 } & CER & SEV & CER & SEV & CER & SEV \\
\hline$f_{7}, f_{8}, f_{17}$ & $0 \%$ & 0.2 & $100 \%$ & 1.5 & $33 \%$ & 0.2 \\
\hline
\end{tabular}

three causes give the $50 \%$ chances of having individual causes. This is realistic also because the factor belongs to all the three categories. Test Case 2: Considering the patient observed to have $f_{7}, f_{8}, f_{17}$ is shown in Table 6 The patient is suffering from pneumonia as he is having the problem of a cough with mucus or even blood. Therefore the certainty is coming to be $100 \%$. Also, the factors $f_{7}, f_{8}$ are dominant in pneumonia and hence showing high severity.

The introduced cognitive approach for inference diagnosis problem in a patient with expert system method ensures no use of old-style rule-based inference mechanisms but computes the certainty and severity of suspected causes using the participation ratio value and expert confidence value respectively.

\section{CONCLUSION}

Providing expert system services using cognitive approach has several advantages, especially for the medical domain. Developing applications for an expert system with user interaction platforms is not as easy considering its limited capabilities. So to avoid these limitations, other researchers have adopted the client-server knowledge-based architecture for developing open expert system applications, where only the interface realized on the user side, and the core application executed on a remote server. While this idea has many benefits including ease of maintenance, upgrades, portable and easy deployment mechanism, building a standalone expert system on cognitive inference surpasses these leads to accessibility. This additional advantage is essential for medical diagnostic expert systems. As a research problem, the work deliberated the contests that might challenge with, on our way to constructing standalone expert system applications. The work formulated a modest knowledge representation for showing knowledge based on XML language, a user interface and an inference mechanism for that purpose. Also, the paper presented a diagnosis problemsolving method that supports human-like cognitive inference mechanism to handle multiple-factor supposition by providing a scientific assessment of the relationship between factors and causes in the form of expected certainty and severity of the supposed disorders based on pragmatic considerations. The proposed model is established efficiently and verified using real knowledge base for the Fever. The attained consequences disclose the efficacy of the planned problem solving based on the cognitive model, as it does not rely on the traditional rule-based and internet search methods that usually used in expert system applications. It also needs massive amount of storing and handling power, but it practices precalculated certainty and severity factors for each factor/cause combination, and arrange the results according to the calculated values for each disorder. In the Future work, the diagnosis will be based on more factors depending on external and internal factors related to patients.

\section{REFERENCES}

[1] Janice S Aikins, John C Kunz, Edward H Shortliffe, and Robert J Fallat. Puff: an expert system for interpretation of pulmonary function data. Computers and biomedical research, 16(3):199-208, 1983.

[2] Mohammad-R Akbarzadeh-T and Majid MoshtaghKhorasani. A hierarchical fuzzy rule-based approach to aphasia diagnosis. Journal of Biomedical Informatics, 40(5):465-475, 2007.

[3] Elias M Awad and Dustin Huntington. Building expert systems: principles, procedures, and applications. West Publishing Co., 1996.

[4] Fatih Başçiftçi and Hayri İncekara. Web based medical decision support system application of coronary heart disease diagnosis with boolean functions minimization method. Expert Systems with Applications, 38(11):14037-14043, 2011.

[5] I Bonfa, C Maioli, F Sarti, GL Milandri, and PR Dal Monte. Hermes: an expert system for the prognosis of hepatic diseases. In Artificial Neural Networks and Expert Systems, 1993. Proceedings., First New Zealand International TwoStream Conference on, pages 240-246. IEEE, 1993.

[6] Aicha Boutorh and Ahmed Guessoum. Complex diseases snp selection and classification by hybrid association rule mining and artificial neural networkbased evolutionary algorithms. Engineering Applications of Artificial Intelligence, 51:58-70, 2016.

[7] Ashish Chandiok and DK Chaturvedi. Cognitive decision support system for medical diagnosis. In Computational Tech- 
niques in Information and Communication Technologies (ICCTICT), 2016 International Conference on, pages 337-342. IEEE, 2016.

[8] Wei-Ling Chen, Chung-Dann Kan, Chia-Hung Lin, and Tainsong Chen. A rule-based decision-making diagnosis system to evaluate arteriovenous shunt stenosis for hemodialysis treatment of patients using fuzzy petri nets. IEEE Journal of Biomedical and Health Informatics, 18(2):703-713, 2014.

[9] Michael Cherkassky. Application of machine learning methods to medical diagnosis. Chance, 22(1):42-50, 2009.

[10] Yu-Liang Chi, Tsang-Yao Chen, and Wan-Ting Tsai. A chronic disease dietary consultation system using owl-based ontologies and semantic rules. Journal of biomedical informatics, 53:208-219, 2015.

[11] Nassim Douali, Huszka Csaba, Jos De Roo, Elpiniki I Papageorgiou, and Marie-Christine Jaulent. Diagnosis support system based on clinical guidelines: comparison between casebased fuzzy cognitive maps and bayesian networks. Computer methods and programs in biomedicine, 113(1):133143, 2014.

[12] Francesco Gagliardi. Instance-based classifiers applied to medical databases: diagnosis and knowledge extraction. Artificial intelligence in medicine, 52(3):123-139, 2011.

[13] Joseph C Giarratano and Gary Riley. Expert systems: principles and programming. Brooks/Cole Publishing Co., 1989.

[14] Donna L Hudson and Maurice E Cohen. Fuzzy logic in medical expert systems. IEEE Engineering in Medicine and Biology Magazine, 13(5):693-698, 1994.

[15] Borna Jafarpour, Samina Raza Abidi, and Syed Sibte Raza Abidi. Exploiting semantic web technologies to develop owlbased clinical practice guideline execution engines. IEEE journal of biomedical and health informatics, 20(1):388-398, 2016.

[16] Ali Keleş and Aytürk Keleş. Estdd: Expert system for thyroid diseases diagnosis. Expert Systems with Applications, 34(1):242-246, 2008.

[17] Ali Keleş, Aytürk Keleş, and Uğur Yavuz. Expert system based on neuro-fuzzy rules for diagnosis breast cancer. $E x$ pert systems with applications, 38(5):5719-5726, 2011.

[18] K Ashwin Kumar, Yashwardhan Singh, and Sudip Sanyal. Hybrid approach using case-based reasoning and rule-based reasoning for domain independent clinical decision support in icu. Expert Systems with Applications, 36(1):65-71, 2009.

[19] Chang-Shing Lee and Mei-Hui Wang. A fuzzy expert system for diabetes decision support application. IEEE Transactions on Systems, Man, and Cybernetics, Part B (Cybernetics), 41(1):139-153, 2011.

[20] Chunhong Lu, Zhaomin Zhu, and Xiaofeng Gu. An intelligent system for lung cancer diagnosis using a new genetic algorithm based feature selection method. Journal of medical systems, 38(9):97, 2014.

[21] P Mohapatra, Sreejit Chakravarty, and Pradipta K Dash. An improved cuckoo search based extreme learning machine for medical data classification. Swarm and Evolutionary Computation, 24:25-49, 2015.

[22] Elpiniki I Papageorgiou. A new methodology for decisions in medical informatics using fuzzy cognitive maps based on fuzzy rule-extraction techniques. Applied Soft Computing, 11(1):500-513, 2011.
[23] Matthias Samwald, Jose Antonio Miñarro Giménez, Richard D Boyce, Robert R Freimuth, Klaus-Peter Adlassnig, and Michel Dumontier. Pharmacogenomic knowledge representation, reasoning and genome-based clinical decision support based on owl $2 \mathrm{dl}$ ontologies. BMC medical informatics and decision making, 15(1):12, 2015.

[24] Rainer Schmidt, Stefania Montani, Riccardo Bellazzi, Luigi Portinale, and Lothar Gierl. Cased-based reasoning for medical knowledge-based systems. International Journal of Medical Informatics, 64(2-3):355-367, 2001.

[25] Edward Shortliffe. Computer-based medical consultations: MYCIN, volume 2. Elsevier, 2012.

[26] Edward H Shortliffe. Medical expert systemsknowledge tools for physicians. Western Journal of Medicine, 145(6):830, 1986.

[27] Ruxandra Stoean and Catalin Stoean. Modeling medical decision making by support vector machines, explaining by rules of evolutionary algorithms with feature selection. Expert Systems with Applications, 40(7):2677-2686, 2013.

[28] William van Melle, Edward H Shortliffe, and Bruce G Buchanan. Emycin: A knowledge engineers tool for constructing rule-based expert systems. Rule-based expert systems: The MYCIN experiments of the Stanford Heuristic Programming Project, pages 302-313, 1984.

[29] Xiaowei Zhang, Bin $\mathrm{Hu}, \mathrm{Xu}$ Ma, Philip Moore, and Jing Chen. Ontology driven decision support for the diagnosis of mild cognitive impairment. Computer methods and programs in biomedicine, 113(3):781-791, 2014.

[30] Yi-Fan Zhang, Ling Gou, Yu Tian, Tian-Chang Li, Mao Zhang, and Jing-Song Li. Design and development of a sharable clinical decision support system based on a semantic web service framework. Journal of medical systems, 40(5):118, 2016. 